\title{
Localized Gingival Enlargement induced by Systemic Administration of Phenytoin for the Management of Epidermolysis Bullosa
}

\author{
${ }^{1} \mathrm{~K}$ Smitha Gowda, ${ }^{2}$ Shilpa Vinod
}

\begin{abstract}
Epidermolysis bullosa (EB) represents a spectrum of diseases that are characterized by increased fragility and blistering of the skin. At present, while there is no specific treatment for $E B$, the therapeutic approaches are essentially palliative, which aims at achieving an acceptable quality of life. A rare case of EB in a 15-year-old Indian boy who was on phenytoin for its proposed action on collagenase and who developed localized gingival overgrowth as a side effect is reported here. The patient presented with vesiculobullous lesion, anonychia, dental anomalies and high caries activity which are the clinical manifestations of EB.
\end{abstract}

Keywords: Phenytoin, Gingival overgrowth, Epidermolysis bullosa, Enamel hypoplasia, Dental caries, Oral manifestations.

How to cite this article: Gowda KS, Vinod S. Localized Gingival Enlargement induced by Systemic Administration of Phenytoin for the Management of Epidermolysis Bullosa. Int $\mathrm{J}$ Experiment Dent Sci 2015;4(1):58-61.

Source of support: Nil

Conflict of interest: None

\section{INTRODUCTION}

Epidermolysis bullosa (EB) is a group of hereditary diseases characterized by blistering of tissues lined by stratified epithelia. ${ }^{1,2}$ Based on the level of blistering within the dermal-epidermal junction, they have been classified into four types, namely: EB simplex (EBX), junctional EB (JEB) and dystrophic EB (DEB), which comprises dominant and recessive variants, and Kindler syndrome, which is a mixed level of cleavage. The available literature review has suggested the incidence of EB to be between 1:50,000 and 1:500,000 live birth, worldwide, affecting all racial and ethnic groups. Epidermolysis bullosa affects

\footnotetext{
${ }^{1}$ Reader, ${ }^{2}$ Assistant Professor

${ }^{1}$ Department of Periodontics, Government Dental College and Research Institute, Bengaluru, Karnataka, India

${ }^{2}$ Department of Skin and Venereal Disease, Bangalore Medical College and Research Institute, Bengaluru, Karnataka, India

Corresponding Author: K Smitha Gowda, Reader, Department of Periodontics, Government Dental College and Research Institute, Bengaluru, Karnataka, India, Phone: 9449630572 e-mail: periosmitha@gmail.com
}

both the sexes equally, often manifesting at birth or during first year of life. ${ }^{3,5}$ The range of severity of EB lesions can vary from minor blistering through to severe, massive blistering resulting in scarring which may result in:

- Breathing or swallowing difficulties

- Malnutrition due to feeding difficulties

- Nail changes

- Scarring of the involved areas which may result in difficulties in movement

- Skin atrophy at the site of blisters

- Skin cancer

- Vision impairment

- Death

There is currently no specific treatment for EB, but only palliative treatment, which can help to ease and control symptoms. The main goal of treatment in EB is to avoid skin damage, to minimize the risk of developing infection and malnutrition, to ease the discomfort of the patient and to improve the quality of life. This often requires multidisciplinary approach and needs the dedicated team of a dermatologist, a dentist, a dietician and a physiotherapist.

Currently, research works are being carried out to find better ways to manage EB which include gene replacement therapy, bone marrow transplantation and recombinant protein therapies. However, these are still in their infancy. Phenytoin, a commonly prescribed anticonvulsant for the management of grand mal and psychomotor epilepsy, has been tried in the field of dermatology. It has been used to treat ulcers, epidermolysis bullosa and inflammatory conditions for its proposed mechanism of action on collagenase. ${ }^{6}$ The topical application of phenytoin has shown to promote wound healing and appears to be promising. However, it requires further clinical trials for the evaluation of its efficacy for topical application. The morbidity associated with its side effects following systemic administration is quite common and they include gingival enlargement, coarsening of facies and hirsutism. Some of the other, not so common side effects include drug-induced lupus, purple-hand syndrome, pigmentary alterations and IgA bullous dermatosis. Apart from these side effects, it can interfere with clotting mechanism and can alter folic acid 
and mineral levels in the blood. Administration of phenytoin in pregnant women can cause spectrum of structural, developmental, and behavioral changes known as the fetal hydantoin syndrome in the fetus.

Here, we report a case of EB, who was on oral phenytoin and subsequently developed localized gingival enlargement as its side effect and the clinical manifestations of EB.

\section{CASE REPORT}

A 15-year-old boy who was diagnosed as having recessive dystrophic EB (RDEB) was referred to Department of Periodontics, Government Dental College and Research Institute, Bengaluru, for the evaluation of gingival enlargement by dermatologist (SV). Informants being the parents, the boy was born out of second degree consanguineous marriage, was diagnosed with EB since the age of 1 year. There is history of on and off vesicles and bullous lesions since then, which were treated with topical medication. Phenytoin in the dosage of $10 \mathrm{mg} / \mathrm{kg}$ per day was administered since 5 months for the management of dermal lesions. No other medication is used except palliative topical medication.

There is no history of similar complaint in the family and siblings. The child's developmental milestones were normal. Cutaneous examination revealed multiple vesicles and bullous lesions of size ranging from 0.5 to $2 \mathrm{~cm}$ in diameter distributed mainly on the extremities. Surrounding skin shows scarring with atrophy and pigmentation due to healing of old lesions (Fig. 1). There was loss of fingernails and toenails and his hands showed some degree of deformity due to scarring.

Intraoral examination revealed abundant plaque accumulation with PI being 1.5, (Fig. 2) Enamel hypoplasia with pitting of the surface of the enamel was noted. No vesiculobullous lesion was noted in the oral cavity. All the permanent teeth up to second molar except 13 were

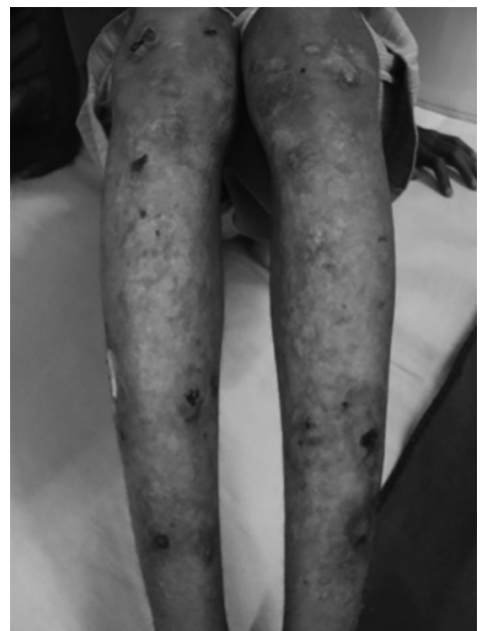

Fig. 1: Vesiculobullous lesions on the legs

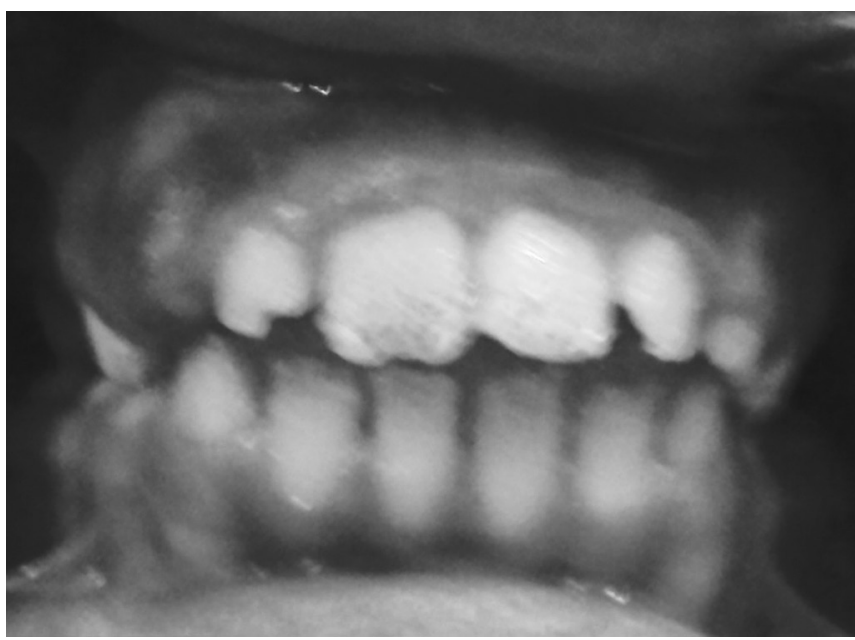

Fig. 2: Intraoral photograph showing the enamel defect and altered contour of the gingiva

present in the oral cavity. Dental decay was present in relation to 16,17 and 26,36, 37, 46. Patient reported that 13 had not erupted and had noticed gingival enlargement in relation to 23 since 2 months (Figs 3 and 4). On examination, the tooth \#23 had erupted and had reached the line

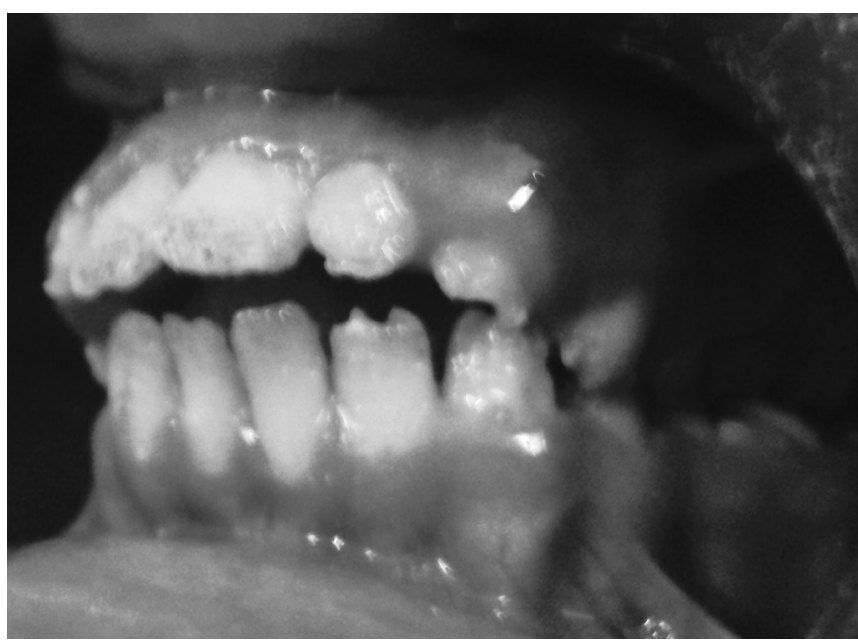

Fig. 3: Intraoral photograph showing enlargement of gingiva in relation to \#23

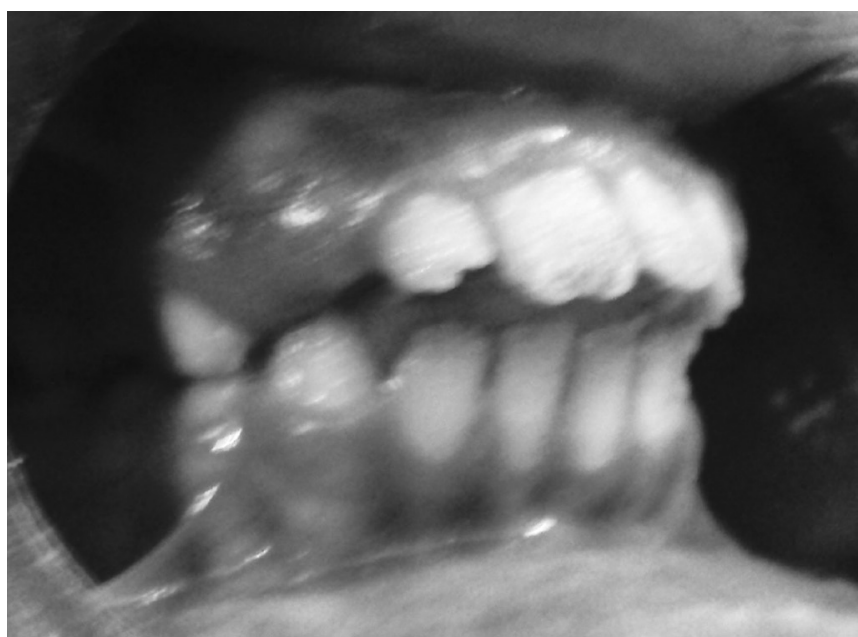

Fig. 4: Intraoral photograph showing clinically missing \#13 
of occlusion. A sizeable gingival enlargement was seen extending from the distal surface of 22 to mesial surface of 24 with the consistency of gingival being firm and resilient. Change in contour of gingival was also noted in relation to maxillary anterior teeth, with rounded of the marginal gingiva. Radiological examination could not be carried out considering the acute nonambulatory condition of the patient in that visit and was postponed till the next appointment though the patient did not return for the next appointment.

\section{MANAGEMENT}

Initially, patient was poorly cooperative and did not permit even simple dental examination, owing to his acute condition. It required a lot of efforts in convincing and counseling the patient to cooperate in performing preliminary dental examination. Preventive measures, which comprised elementary instruction on oral health care, diet counseling and improvement of oral care skills by means of training in tooth brushing and flossing were administered $0.12 \%$. Chlorhexidine mouth wash was prescribed and was instructed to use twice daily. Informed written consent was taken from parents of the patient for reporting and for management of the case. Treatment plan consisting of periodontal and restorative treatment was explained to the patient, and the patient was discharged with the instruction to return for further dental treatment once the acute dermal condition subsided. However, patient has failed to comply with the proposed treatment plan and has not returned for further dental treatment for unknown reasons.

\section{DISCUSSION}

This case is remarkable not only because very few cases have been reported describing the oral manifestation of this rare disease but also because no report so far has described the phenytoin induced gingival enlargement which was used in the management of EB, though the literature is replete with reports describing the phenytoin induced gingival enlargement when used as a anticonvulsant.

The dental treatment approach for patients with EB, especially for the one with most severe type, has changed by leaps and bounds in the last three decades. While Crawford et $\mathrm{al}^{7}$ in 1976 advised more radical approach of extraction of all teeth as a treatment of choice for EB; two decades later in 1999, Wright $^{8}$ recommended combination of anesthetic and restorative technique for the successful management of dental abnormalities. More recently, Krämer et $\mathrm{al}^{9}$ has shown that caries can be successfully prevented in patients with RDEB by regular follow-up of patients aiming at dietary management to prevent malnutrition, oral hygiene habits, frequent professional cleaning and fluoride application. Several studies have shown that phenytoin is useful in treating recessive dystrophic epidermolysis bullosa and in reducing its blister count. 10,11 Such, efficacy has been noted in case reports of single patients in Taiwan ${ }^{12}$ and Italy. ${ }^{13}$ It has been used in short- and long-term management of dystrophic epidermolysis bullosa. ${ }^{14}$ In the present case, EB was managed with administration of phenytoin. The localized gingival enlargement could be attributed to phenytoin administration as the patient noticed gingival enlargement only since 2 months and was on phenytoin since 5 months.

The teeth in the present case showed enamel hypoplasia, with pitting of the surface, further complicating the plaque removal. The effect of EB on tooth formation and structure is unclear, and its clinical expression is highly variable. ${ }^{1}$ The severity of abnormalities of the dental enamel range from mild pit defects to severe hypoplasia, thin enamel or loss of enamel. ${ }^{15-17}$ However, Liversidge et $\mathrm{al}^{18}$ are of the opinion that permanent tooth maturation proceeds normally in children with DEB. The present case showed clinically missing 13, though the agenesis of 13 could not be ascertained as the radiograph was not available. However, the patient did not give any history of extraction of the particular tooth. Lindemeyer et $\mathrm{al}^{19}$ reported a patient with congenitally missing maxillary right lateral incisor.

Wright et $\mathrm{al}^{20}$ have found higher prevalence of caries among EB individual than among healthy individuals. Excessive caries has been suggested to be due to the presence of severe soft-tissue involvement, which leads to alteration of diet, increased oral clearance rate, creation of an abnormal tooth/soft-tissue relationship. This complicates the oral hygiene measures leading to higher caries activity. The present case reports high caries activity.

This case report is limited by lack of radiographic report and histopathological report suggestive of druginduced enlargement. However, there is positive history of phenytoin administration in therapeutic doses and concomitant development of gingival overgrowth and histopathological changes in drug-induced enlargement is nonspecific.

\section{SUMMARY}

Epidermolysis bullosa is a rare hereditary disease with multiple oral manifestations. The management of these oral manifestations requires special approach, often requiring interdisciplinary approach of a dentist, a dermatologist and a dietician, which is emphasized in the present 
case. Dentists should be aware of the clinical presentation of EB for the early diagnosis and to give primary preventive and restorative care. Prevention of caries and periodontal disease must be emphasized at a very young age as it is very difficult to manage the dental problems at a later stage in adulthood. Dentistry is varying with induction of modern science to practice dentistry. ${ }^{21}$

\section{REFERENCES}

1. Fine JD, Eady RA, Bauer EA, Bauer JW, Bruckner-Tuderman L, Heagerty A, et al. The classification of inherited epidermolysis bullosa (EB): report of the Third International Consensus Meeting on Diagnosis and Classification of EB. J Am Acad Dermatol 2008;58:931-950.

2. Stavropoulos F, Abramowicz S. Management of the oral surgery patient diagnosed with epidermolysis bullosa: report of 3 cases and review of the literature. J Oral Maxillofac Surg 2008 Mar;66(3):554-559.

3. Pacheco W, Marques de Sousa Araugio R. Orthodontic treatment of a patient with recessive dystrophic epidermolysis bullosa: a case report. Spec Care Dentist 2008;28(4):136-139.

4. Prabhu VR, Rekka P, Ramesh, Swathi S. Dental and anesthetic management of a child with epidermolysis bullosa. J Ind Soc Pedod Prev Dent 2011;29(2):155-160.

5. Silva IG, Alves JMA, Mattos LMBB, Francisquini RF. Epidermolysis bullosa. HU Rev 2000;26:221-227.

6. Eugene A, Bauer MD, Thomas W, Cooper MD, Dolores R, Tucker MD, et al. Phenytoin therapy of recessive dystrophic epidermolysis bullosa-clinical trial and proposed mechanism of action on collagenase. N Engl J Med 1980 Oct;303:776-781.

7. Crawford EG Jr, Burkes EJ Jr, Briggaman R. A hereditary epidermolysis bullosa: oral manifestations and dental therapy. Oral Surg Oral Med Oral Pathol 1976 Oct;42(4):490-500.

8. Wright JT. Oral manifestations of epidermolysis bullosa. In: Fine JD, Bauer EA, McGuire J, Moshell A, editors. Epidermolysis bullosa: clinical, epidemiologic and laboratory advances and the findings of the National Epidermolysis
Bullosa Registry. Baltimore: Johns Hopkins University Press. 1999. p. 236-257.

9. Krämer SM, Serrano MC, Zillmann G, Gálvez P, Araya I, Yanine N, et al. Oral healthcare for patients with epidermolysis bullosa-best clinical practice guidelines. Int J Paediat Dentist 2012; 22 (Suppl 1):1-35.

10. Scheinfeld N. Phenytoin in cutaneous medicine: its uses, mechanisms and side effects. Dermatol Online J 2003 Aug; 9(3):6.

11. Abahussein AA, al-Zayir AA, Mostafa WZ, Okoro AN. Epidermolysis bullosa in the eastern province of Saudi Arabia. Int J Dermatol 1993 Aug;32(8):579-581.

12. Yen SL, Chao LH, Ho MM, Hwang KC. Epidermolysis bullosa: report of one case. Zhonghua Min Guo Xiao Er Ke Yi Xue Hui Za Zhi 1990 Nov-Dec;31(6):383-387.

13. Altomare GF, Polenghi M, Pigatto PD, Nazzaro V, Piattoni F. Dystrophic epidermolysis bullosa inversa. Dermatologica 1990;181(2):145-148.

14. Cooper TW, Bauer EA. Therapeutic efficacy of phenytoin inrecessive dystrophic epidermolysis: a comparison of shortand long-term treatment. Arch Dermatol 1984 Apr;120(4): 490-495.

15. Wright JT, Johnson LB, Fine JD. Development defects of enamel in humans with hereditary epidermolysis bullosa. Arch Oral Biol 1993 Nov;38(11):945-955.

16. Wright JT, Gantt DG. Epidermolysis bullosa associated with enamel hypoplasia and taurodontism. J Oral Pathology 1983;12(2):73-83.

17. Wright JT. Oral manifestations in the epidermolysis bullosa spectrum. Dermatol Clin 2010;28(1):159-164.

18. Liversidge HM, Kosmidou A, Hector MP, Roberts GJ. Epidermolysis bullosa and dental developmental age. Int J Paediatr Dent 2005 Sep;15(5):335-341.

19. Lindemeyer R, Wadenya R, Maxwell L. Dental and anaesthetic management of children with dystrophic epidermolysis bullosa. Int J Paediatr Dent 2009 Mar;19(2):127-134.

20. Wright JT, Fine JD, Johnson L. Dental caries risk in hereditary epidermolysis bullosa. Pediatr Dent 1994;16(6):427-432.

21. Saini R. Ozone therapy in dentistry: a strategic review. J Nat Sc Biol Med 2011;2(2):151-153. 